\title{
Some fundamental properties for regular element of electroencephalography signals semigroup during epileptic seizure
}

\author{
Ameen Omar Ali Barja ${ }^{1}$, Tahir Ahmad ${ }^{1, *}$, Faisal Abdurabu Mubarak Binjadhnan ${ }^{2}$
}

${ }^{1}$ Department of Mathematical Science, and Ibnu Sina Institute for Fundamental Science Studies, Nanotechnology Research Alliance, Faculty of Science Universiti Teknologi Malaysia 81310 UTM Johor Bahru, Johor, Malaysia.

${ }^{2}$ Department of Mathematics, Faculty of science, Hadhramout University, Mukalla 50511, Yemen.

*Corresponding Author: tahir@ibnusina.utm.my

\section{Article history :}

Received 9 September 2014

Accepted 20 February 2015

GRAPHICAL ABSTRACT

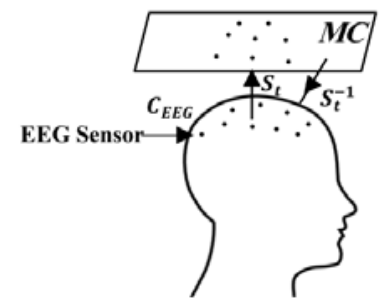

\section{ABSTRACT}

Electroencephalography (EEG) is a record of electrical activity along the scalp. It is measures voltage fluctuations resulting from ionic current flows within the neurons of the brain. EEG is most often used to diagnose epilepsy, which causes understandable abnormalities in EEG readings. The mathematical analysis of EEG signals assists medical specialists by providing a clarification of the brain activity being observed, so increasing the information about the structure and function of the human brain. EEG signals during epileptic seizure can be viewed as a semigroup of square matrices under matrix multiplication, and every element in that semigroup is shown to be regular. In this paper, we will present some main properties of regular element of EEG signals during epileptic seizure.

Keywords: EEG, semigroup, regular element

\section{INTRODUCTION}

Epilepsy is a group of long term neurological disorders categorized by seizures. These seizures are episodes that can differ from brief and nearly undetectable to long periods of strong trembling. In epilepsy, seizures tend to recur, and have no direct underlying cause while seizures that occur due to an exact cause are not deemed to represent epilepsy [1]. It is categorized into two major groups, partial and generalized. Partial seizures are those in which the clinical or electroencephalographic evidence recommends that the attacks have a localized onset in the brain [2]. This class of seizure affects only a part of the cerebral hemisphere at seizure onset and produces symptoms in corresponding parts of the body or disturbances in some related mental functions. Perversely, generalized seizures are said to occur if the evidence proposes that the attacks were well spread [3].

An instrument that measures electrical potentials (brain waves) on the scalp and produces a record of the electrical activity of the brain called Electroencephalography (EEG). It is a key tool in the diagnosis and management of epilepsy and further seizure disorders, as well as in the diagnosis of brain damage related to trauma and diseases such as strokes, tumors, encephalitis, drug, and alcohol intoxication. Furthermore, EEG is also useful in control brain wave activity and in the brain death determination [4].

The mathematical analysis of EEG signals may assist the medical professionals by providing an illustration of the brain activity being observed, therefore increasing the understanding of the brain function of
(C) 2015 Penerbit UTM Press. All rights reserved http://dx.doi.org/10.11113/mjfas.v11n1.335 human. Up to the moment, the Fast Fourier Transform (FFT) is the most used quantitative technique for EEG signals analysis. However, it has some disadvantages that limit its applicability. Hence, further methods for extracting "hidden" information from the EEG signals are required.

\section{FLATTENING THE EEG SIGNALS}

A new technique to map high dimensional signal, namely EEG into low dimensional space had been developed by Zakaria and Ahmad in 2007 [5]. The procedure of this technique contained three important sections. The first one deals with flattening the EEG data which essentially entails transformation of threedimensional spaces into two-dimensional spaces. This procedure contains the position of sensors on the patient's head with EEG signal. The second section comprises processing EEG signals via Fuzzy c-Means clustering. The last section contains finding the optimal number of clusters using cluster validity analysis.

The EEG coordinate system (Figure 1(a)) is defined by Zakaria and Ahmad in 2007 as follows: $C_{E E G}=\left\{\left((x, y, z), e_{p}\right): x, y, z, e_{p} \in \mathbb{R}\right.$ and $\left.x^{2}+y^{2}+z^{2}=r^{2}\right\}$ where $r$ is the radius of a patient head [5]. In addition, a function is defined from $C_{E E G}$ to $M C$ plan as the following: $S_{t}: C_{E E G} \rightarrow M C$ (Figure 1 (b)) such that $S_{t}\left((x, y, z), e_{p}\right)=\left(\frac{r x+i r y}{r+z}, e_{p}\right)=$ $\left(\frac{r x}{r+z}, \frac{r x}{r+z}\right)_{e_{p}(x, y, z)}$

Both, $C_{E E G}$ and $M C$ were designed and verified as 2-manifolds [6]. In this situation, it must be well-known that $S_{t}$ is an injective mapping of a conformal structure. 
Therefore, $S_{t}$ can preserve information in a particular angle and orientation of the surface through the recorded EEG signals. In addition, they implemented this method followed by clustering on real time EEG data obtained from patients who suffer from seizure.

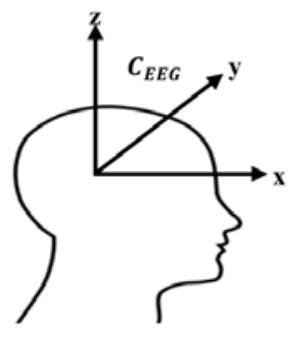

(a)

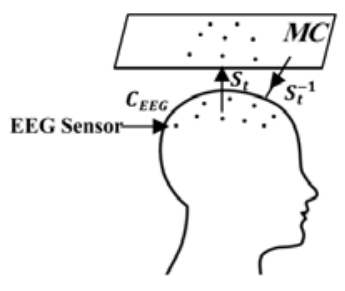

(b)
Figure 1. (a) EEG coordinate system (b) EEG projection

The signals (Figure 2) were digitized at 256 samples per second using Nicolet One EEG software. The average potential difference was calculated from the 256 samples of raw data at every second. Similarly to the location of the electrodes, the EEG signal was preserved through this new method. Every single second of the particular average potential difference was stored in a file which contains the position of the electrode on $M C$ plane (Figure 3).

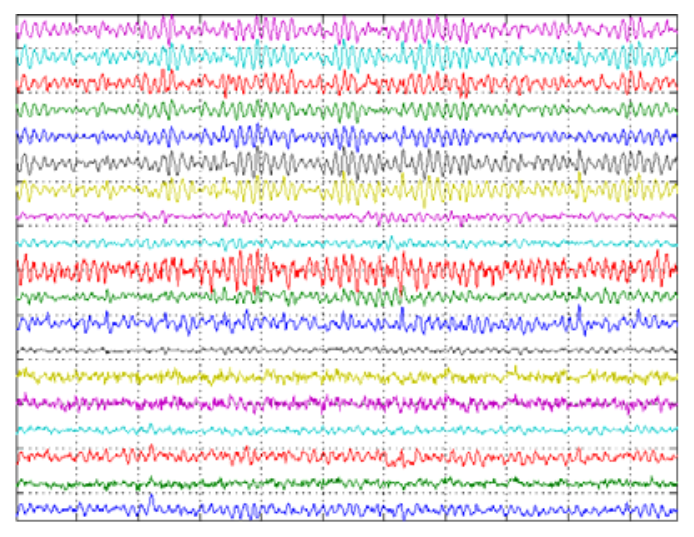

Figure 2. Sample of EEG signal

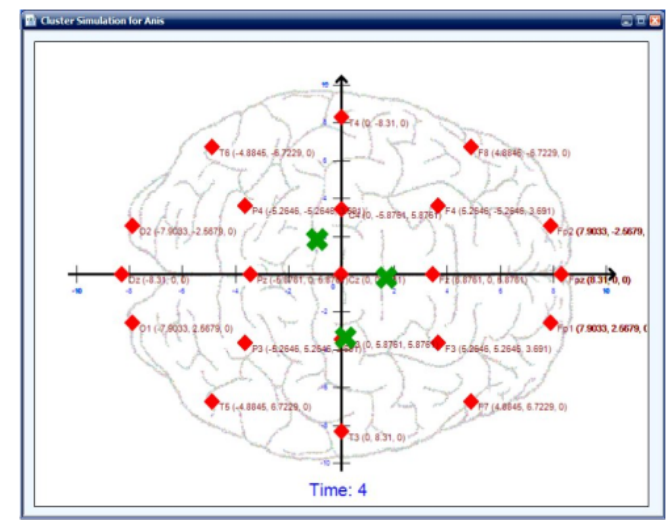

Figure 3. Flat EEG signal

\section{SEMIGROUP OF EEG SIGNALS DURING EPILEPTIC SEIZURE}

Binjadhnan and Ahmad (2010) [7] shown EEG signals during epileptic seizure can be composed into a set of $n \times n$ square matrices

$$
M C_{n}(\mathbb{R})=\left\{\left[\beta_{i j}(z)_{t}\right]_{n \times n}: i, j \in \mathbb{Z}^{+}, \beta_{i j}(z)_{t} \in \mathbb{R}\right\}
$$

where, $\beta_{i j}(z)_{t}$ is a potential difference reading of EEG signals from a particular $i j$ sensor at time $t$. In addition, they transformed the set $M C_{n}(\mathbb{R})$ to the set of upper triangular matrices denoted by $M C_{n}^{\prime \prime}(\mathbb{R})$ using $Q R$-real Schur triangularization (Figure 4) as following:

$$
\begin{aligned}
& M C_{n}^{\prime \prime}(\mathbb{R})=\left\{\left[\beta_{i j}(z)_{t}\right]_{n \times n}: \beta_{i j}(z)_{t}=0, \forall 1 \leq j<i \leq\right. \\
& \left.n, i, j \in \mathbb{Z}^{+}, \beta_{i j}(z)_{t} \in \mathbb{R}\right\} .
\end{aligned}
$$

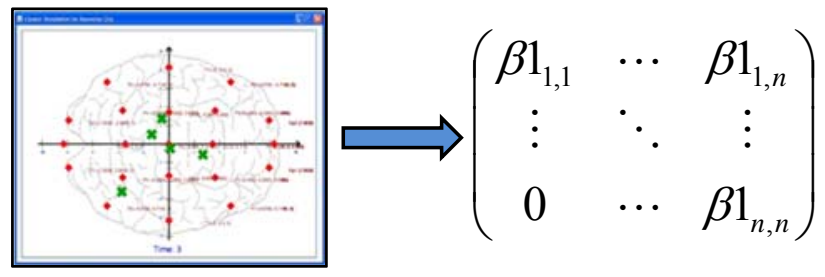

Figure 4. $M C$ plan transformed into an upper triangular matrix

Furthermore, the set $M C_{n}^{\prime \prime}(\mathbb{R})$ satisfies all the axioms of a semigroup under matrix multiplication. Particularly, $M C_{n}^{\prime \prime}(\mathbb{R})$ are closed and associative under matrix multiplication.

\section{MATERIALS AND METHODS}

In this section, some existing definitions and results that will be used throughout this paper are presented.

Definition1 [8]: A subset $\alpha \subseteq\{1,2, \ldots \ldots, n\}$ with the property that for any $i \leq j \leq k \in\{1,2, \ldots \ldots, n\}$ with $i, k \in \alpha$ with $j \in \alpha$ is called $n-$ interval. Let $A$ be $n \times$ $n$ matrix over a field $F$ and $\alpha$ an $n$-interval the restriction $\left.A\right|_{\alpha}$ is the matrix $\left(A_{i j}\right)_{i, j \in \alpha}$ with rows and columns indexed by $\alpha$. Two matrices $A$ and $B$ agree on $\alpha$ if $A_{i j}=B_{i j}$ for all $i, j \in \alpha$. We say that $A$ and $B$ are scalar multiples on $\alpha$ if there exists a non-zero field element $\lambda \in F$ such that $A_{i j}=\lambda B_{i j}$ for all $i, j \in \alpha$, such that $A$ and $\lambda B$ agree on $\alpha$.

Definition2 [9]: Let $S(n, F)$ be a semigroup of all $n \times n$ upper triangular matrices with entries drawn from field $F$, with usual operation (matrix multiplication). Let $A \in$ $S(n, F)$, the diagonal shape of $A$ is the set $\operatorname{shape}(A)=$ $\left\{i \in Z \mid 1 \leq i \leq n, a_{i i} \neq 0\right\}$. Note that two matrices have the same diagonal shape if they have zeros in exactly the same positions on the main diagonal. 
Theorem1 [10]: If $A, B$ are upper triangular matrices. Then $\operatorname{shape}(A B)=\operatorname{shape}(A) \cap \operatorname{shape}(B)$.

Let $A_{t}, B_{t}$ two matrices in $M C_{n}^{\prime \prime}(\mathbb{R})$ and we define a relation $\Omega$ on a semigroups $M C_{n}^{\prime \prime}(\mathbb{R})$ by $A_{t} \Omega B_{t}$ if and only if $A_{t}=\lambda B_{t}$ for some non-zero field element $\lambda$. A relation $\Omega$ on the $M C_{n}^{\prime \prime}(\mathbb{R})$ is called left compatible if $\forall A_{t}, B_{t}, C_{t} \in M C_{n}^{\prime \prime}(\mathbb{R}), A_{t} \Omega B_{t} \Rightarrow C_{t} A_{t} \Omega C_{t} B_{t}$ and right compatible if $\forall A_{t}, B_{t}, C_{t} \in M C_{n}^{\prime \prime}(\mathbb{R}), A_{t} \Omega B_{t} \Rightarrow$ $A_{t} C_{t} \Omega B_{t} C_{t}$ and it is called compatible if $\forall A_{t}, B_{t}, A_{t}^{\prime}, B_{t}^{\prime} \in M C_{n}^{\prime \prime}(\mathbb{R}), A_{t} \Omega A_{t}^{\prime} \quad$ and $\quad B_{t} \Omega B_{t}^{\prime} \Rightarrow$ $A_{t} B_{t} \Omega A_{t}^{\prime} B_{t}^{\prime}$. A left (right) compatible equivalence relation is called a left (right) congruence. A compatible equivalence relation is called congruence.

Definition3 [11]: A row operation on an upper triangular matrix is said to be invertible if we can add a multiple of one row to a row above or scaling a row by non-zero field element.

Remark 1: Column operations are defined analogously.

Let $A_{t}, B_{t} \in M C_{n}^{\prime \prime}(\mathbb{R})$. It is easy to see that $B_{t} A_{t}$ can be obtained from $A_{t}$ by a certain sequence of row operation determined by the matrix $A_{t}$. Conversely, every row operation can be represented as leftmultiplication by a certain triangular matrix. There is an analogous relationship between right-multiplication and column operations.

A direct consequence of these explanations is the following characterization of Green's relations $\mathcal{L}, \mathfrak{R}$ and $\mathcal{J}$ on the semigroups $M C_{n}^{\prime \prime}(\mathbb{R})$.

In 1951 James Alexander Green introduced five equivalence relations that characterize the elements of a semigroup in terms of the principal ideals. These relations are useful for understanding the nature of divisibility in semigroup [The prime decomposition theorem Krohn-Rhodes 1965] . Moreover, Green relations are particularly significant in the study of regular semigroup.

Definition4 [13]: An element $s$ of a semigroup $S$ is called a regular element of $S$ if there is an element $t$ of $S$ such that $S=s t s$ and $S$ is said to be a regular semigroup if every element of $S$ is regular. Note that the regular elements of semigroup of upper triangular matrices are characterized as those matrices whose rank is equal to the number of their non-zero diagonal entries.

If $A_{t}$ is an element of a semigroup $M C_{n}^{\prime \prime}(\mathbb{R})$, the smallest left ideal contain $A_{t}$ is $M_{n}^{\prime \prime}(\mathbb{R}) A_{t} \cup\left\{A_{t}\right\}$, which may conveniently write as $M C_{n}^{\prime \prime} \backslash(\mathbb{R}) A_{t}$, and called it the principal left ideal generated by $A_{t}$. An equivalence relation $\mathcal{L}$ on $M C_{n}^{\prime \prime}(\mathbb{R})$ is defined by the rule that $A_{t} \mathcal{L} B_{t}$ if and only if $A_{t}$ and $B_{t}$ generate the same principal left ideal, in other words, $M C_{n}^{\prime \prime} \backslash(\mathbb{R}) A_{t}=$ $M C_{n}^{\prime \prime} \backslash(\mathbb{R}) B_{t}$. Similarly, define $\mathfrak{R}$ by the rule that $A_{t} \mathfrak{R} B_{t}$ if and only if $A_{t}$ and $B_{t}$ generate the same principle right ideal, in other words, $A_{t} M C_{n}^{\prime \prime}(\mathbb{R})=$ $B_{t} M C_{n}^{\prime \prime}(\mathbb{R})$. Furthermore, define $\mathcal{J}$ by the rule that
$A_{t} \mathcal{J} B_{t} \quad$ if $\quad$ and $\quad$ only $M C_{n}^{\prime \prime} \backslash(\mathbb{R}) A_{t} M C_{n}^{\prime \prime} \backslash(\mathbb{R})=$
$M C_{n}^{\prime \prime} \backslash(\mathbb{R}) B_{t} M C_{n}^{\prime \prime} \backslash(\mathbb{R})$.

Definition5 [14]: The elementary EEG signals is a square matrix of EEG signals reading at time $t$ in terms of one of the following types:

(i) Diagonal matrix (special case sub-identity matrix).

(ii) Unipotent matrix.

(iii) Permutation.

Proposition1 [15]: Let $S(n, F)$ be a semigroup of all $n \times n$ upper triangular matrices with entries drawn from field $F$. Let $A_{1}, A_{2} \in S(n, F)$ then:

(i) $A_{1}, A_{2}$ are $\mathcal{L}$-related precisely if each can be acquired from the other by row operation.

(ii) $A_{1}, A_{2}$ are $\mathfrak{R}$ - related precisely if each can be acquired from the other by column operation.

(iii) $A_{1}, A_{2}$ are $\mathcal{J}$-related precisely if each can be acquired from the other by row and column operation.

Theorem2 [16]: Assume that $A_{t}$ is an upper triangular matrix of EEG signals during epileptic seizure $\left(A_{t} \in\right.$ $\left.M C_{n}^{\prime \prime}(\mathbb{R})\right)$. Then the following are equivalent:

(i) $A_{t}$ is regular;

(ii) Every row (column) in $A_{t}$ is a linear combination of rows (columns) in $A_{t}$ with non-zero diagonal entries;

(iii) $A_{t}$ is $\mathcal{J}$-related to sub-identity.

\section{CHARACTERIZATION OF REGULAR ELEMENTS OF SEMIGROUP OF EEG SIGNALS DURING EPILEPTIC SEIZURE}

The following is a corollary of Theorem 1 which characterize the $\mathcal{J}$-related of regular elements of $M C_{n}^{\prime \prime}(\mathbb{R})$ in terms of diagonal shape of EEG signals matrix.

Corollary 1: Let $A_{t}, B_{t}$ are upper triangular matrices of EEG signals during epileptic seizure $\left(A_{t}, B_{t} \in\right.$ $\left.M C_{n}^{\prime \prime}(\mathbb{R})\right)$ with $A_{t}, B_{t}$ regulars. Both $A_{t}, B_{t}$ are $\mathcal{J}-$ related if and only if they have the same diagonal shape.

Proof.

Let $A_{t}, B_{t} \in M C_{n}^{\prime \prime}(\mathbb{R})$ and $A_{t}, B_{t}$ are -related .

Since the diagonal shape is preserved by invertible row and column operation, and if $A_{t}, B_{t}$ have zeros in exactly the same positions on the main diagonal, then $\mathcal{J}-$ related for both EEG signals matrices $\left(A_{t}\right.$ and $\left.B_{t}\right)$ needed to have the same diagonal shape (by Proposition 1). Conversely, let $A_{t}$ and $B_{t}$ are regular elements and have the same diagonal shape, then $A_{t}$ and $B_{t}$ are $\mathcal{J}-$ related to the unique sub-identity EEG signals matrices with that diagonal shape (by theorem 2). Thus, they are $\mathcal{J}-$ related to each other. $\square$ 
Theorem 3: Assume that $A_{t}, B_{t}$ are upper triangular matrices of EEG signals during epileptic seizure $\left(A_{t}, B_{t} \in M C_{n}^{\prime \prime}(\mathbb{R})\right)$ with $A_{t}$ regular. Then the following are equivalent:

(i) $\operatorname{shape}\left(A_{t}\right) \subseteq \operatorname{shape}\left(B_{t}\right)$;

(ii) $A_{t} B_{t} \mathfrak{R} A_{t}$;

(iii) $A_{t} B_{t} \mathcal{J} A_{t}$

\section{Proof.}

(i) $\Rightarrow$ (ii)

Let $\operatorname{shape}\left(A_{t}\right) \subseteq \operatorname{shape}\left(B_{t}\right)$ where $A_{t}, B_{t} \in M C_{n}^{\prime \prime}(\mathbb{R})$. By applying column operation (Definition 3) for $A_{t}$ we have $A_{t} B_{t}$ can be acquired from $A_{t}$. Therefore, every column of $A_{t}$ is a linear combination of columns with nonzero diagonal entries (by theorem 2). But since $\operatorname{shape}\left(A_{t}\right) \subseteq \operatorname{shape}\left(B_{t}\right)$, then every column of $A_{t}$ will be a combination of columns of $A_{t} B_{t}$ with nonzero diagonal entries. Thus, $A_{t} B_{t} C_{t}=A_{t}$ for some $C_{t}$, which mean $A_{t} B_{t} \Re A_{t}$ (definition of $\Re-$ relation).

(ii) $\Rightarrow$ (iii)

If $A_{t}$ and $B_{t}$ are regular elements and $\mathcal{J}-$ related, then $\operatorname{shape}\left(A_{t}\right)=\operatorname{shape}\left(B_{t}\right)$ which implies to $A_{t} B_{t} \mathcal{J} A_{t}$ (by Corollary 1).

(iii) $\Rightarrow$ (i)

Let $A_{t} B_{t} \mathcal{J} A_{t}$, then

$\operatorname{shape}\left(A_{t}\right)=\operatorname{shape}\left(A_{t} B_{t}\right)$ (by Proposition 1) $=\operatorname{shape}\left(A_{t}\right) \cap \operatorname{shape}\left(B_{t}\right)$ (by theorem 1$)$

Hence, $\operatorname{shape}\left(A_{t}\right) \subseteq \operatorname{shape}\left(B_{t}\right)$. $\square$

\section{CONCLUSION}

The paper presented the use of Green's relations to characterize the regularity of semigroup elements of EEG signals during epileptic seizure.

\section{ACKNOWLEDGEMENTS}

All praise is due only to ALLAH, the lord of the worlds. Ultimately, only ALLAH has given us the strength and courage to proceed with our entire life. The Authors would like to acknowledge their family members for their continuous support and encouragement. The first author would like to acknowledge Hadhramout University too for granting the scholarship during his study.

\section{References}

[1] Fisher, R.S., W.v.E. Boas, W. Blume, C. Elger, P. Genton, P. Lee, and J. Engel, Epileptic seizures and epilepsy: definitions proposed by the International League Against Epilepsy (ILAE) and the International Bureau for Epilepsy (IBE). Epilepsia, 470472 (2005) 46(4)

[2] Gastaut, H., Clinical and Electroencephalographical Classification of Epileptic Seizures. Epilepsia, 102-112 (1970) 11(1).

[3] Ahmad, T., R.A. Fairuz, F. Zakaria, and H. Isa. Selection of a subset of EEG channels of epileptic patient during seizure using PCA. in Proceedings of the 7th WSEAS International Conference on Signal Processing, Robotics and Automation. (2008): World Scientific and Engineering Academy and Society (WSEAS).

[4] Niedermeyer, E. and F.L. Da Silva, Electroencephalography: basic principles, clinical applications, and related fields. (2005): Lippincott Williams \& Wilkins.

[5] Zakaria, F. and T. Ahmad, Tracking The Storm In The Brain., in Presented at Kolokium Jabatan Matematik. (2007): UTM Skudai Johor.

[6] Fauziah, Z., Dynamic Profiling Of EEG Data During Seizure Using Fuzzy Information Space, in Mathematics. (2008), Universiti Teknologi Malaysia,Skudai: Malaysia.

[7] Binjadhnan, F.A.M. and T. Ahmad, EEG Signals during Epileptic Seizure as a Semigroup of Upper Triangular Matrices. American Journal of Applied Sciences, (2010) 7(4).

[8] Putcha, M.S., Linear algebraic monoids. Vol. 133. (1988): Cambridge University Press.

[9] Almeida, J., S.W. Margolis, and M.V. Volkov, The pseudovariety of semigroups of triangular matrices over a finite field. Theoretical Informatics and Applications, 31-48 (2005) 39(1).

[10] Kambites, M., On the Krohn-Rhodes complexity of semigroups of upper triangular matrices. International Journal of Algebra and Computation, 187-201 (2007) 17(01).

[11] Clifford, A.H. and G.B. Preston, The algebraic theory of semigroups. 2. Vol. 7. (1967): Amer Mathematical Society.

[12] Krohn, K. and J. Rhodes, Algebraic theory of machines. I. Prime decomposition theorem for finite semigroups and machines. Transactions of the American Mathematical Society, 450-464 (1965) 116

[13] Howie, J.M., Fundamentals of semigroup theory. (1995): Clarendon Oxford.

[14] Binjadhnan, F.A.M., Krohn-Rhodes Decomposition for Electroencephalography Signals during Epileptic Seizure., in Mathematics. (2011), Universiti Teknologi Malaysia, Skudai: Malaysia.

[15] Kambites, M. and B. Steinberg, Wreath Product Decompositions for Triangular Matrix Semigroups. Semigroups and Formal Languages, 129-144 (2005).

[16] Barja, A.O., T. Ahmad, and F.A. Binjadhnan, Regular Element for a Semigroup of Electroencephalography Signals During Epileptic Seizure. Journal of Applied Sciences, 1781-1785 (2014) 14(15). 